\title{
ZEUS Electro-Weak Fit and Measurement of the Charged-Current DIS Cross Section
}

\author{
Kunihiro Nagano \\ On behalf of the ZEUS Collaboration \\ HIGH ENERGY ACCELERATOR RESEARCH ORGANISATION, KEK \\ 1-1 Oho, Tsukuba, Ibaraki 305-0801 Japan \\ Email: knagano@post.kek.jp
}

\begin{abstract}
The cross sections for deep inelastic scattering in $e^{ \pm} p$ collisions with longitudinally polarised lepton beams have been measured by the ZEUS experiment at HERA. The polarisation dependence of the charged-current cross sections was examined as a direct means of validating the helicity structure of the weak interaction. In addition, an electro-weak and next-to-leading order QCD analysis has been performed, and the weak neutral vector and axial-vector couplings of quarks have been precisely determined.
\end{abstract}

\section{Introduction}

Deep inelastic scattering (DIS) of leptons off nuclei has been an essential experimental tool in formulating both the electro-weak (EW) and QCD sectors of the Standard Model (SM). HERA at DESY is the first $e^{ \pm} p$ collider, and has significantly extended the kinematic region to explore the proton structure. Based on about $100(20) \mathrm{pb}^{-1}$ of unpolarised $e^{+} p\left(e^{-} p\right)$ data collected until the year 2000 (HERA-I), the ZEUS experiment at HERA has measured the inclusive neutral- and charged-current (NC and CC, respectively) DIS cross sections and the jet-production cross sections in DIS and photoproduction reactions. The wide variety and kinematic range covered by these data, as well as their precision, has allowed the determination of parton distribution functions (PDFs) from HERA-data alone (ZEUSJETS PDFs) [1]. The DGLAP-evolution based perturbative QCD was found to give an excellent description over 5 orders of magnitude in the four-momentum transfer squared, $Q^{2}$, and 3 orders of magnitude in the Bjorken scaling variable, $x$. In particular, the PDFs of gluon and sea quarks were precisely determined in small $x$ region of $10^{-4} \lesssim x \lesssim 10^{-2}$.

After the year 2000, HERA underwent a major upgrade (HERA-II) aiming for higher luminosity and for collisions with longitudinally polarised $e^{ \pm}$beams. This provides not only increased statistics at large $Q^{2}$ where EW contributions dominate but also a direct sensitivity to the helicity structure of the EW interaction. Also, the increased statistics bring more sensitivity to PDFs at large $x$. ZEUS has measured the NC and CC DIS cross sections with polarised $e^{ \pm}$beams provided until the year 2007 when HERA terminated its operation. In addition, a combined EW and QCD analysis was performed to determine both PDFs and EW parameters simultaneously by exploiting these polarised HERA-II data. In this report, the ZEUS measurement of the polarised CC DIS cross sections and the results of the combined EW and QCD analysis are presented.

\section{Polarised $e^{ \pm} p$ CC DIS}

As a direct consequence of the fully parity-violating nature of the weak $\mathrm{CC}$ interaction in the SM, the cross section for CC DIS at HERA, $e^{+(-)} p \rightarrow \bar{\nu}(\nu) X$, depends linearly on the 
longitudinal polarisation of $e^{ \pm}, P_{e}$, as

$$
\sigma_{C C}^{P, \pm}=\left(1 \pm P_{e}\right) \sigma_{C C}^{0, \pm},
$$

where $\sigma_{C C}^{0, \pm}$ denotes the cross section for unpolarised $e^{ \pm} p$ DIS. Polarisation, $P_{e}$, is defined as $\left(N_{R}-N_{L}\right) /\left(N_{R}+N_{L}\right)$, where $N_{R}\left(N_{L}\right)$ denotes the number of right-handed (left-handed) $e^{ \pm}$.

The total CC cross section, corrected to the Born level in the EW interaction, was measured for the kinematic region of $Q^{2}>200 \mathrm{GeV}^{2}$ by using $78.8(42.7) \mathrm{pb}^{-1}$ of $e^{-} p$ collisions at a mean polarisation of $-0.27(+0.33)[2]$, and $79.3(58.9) \mathrm{pb}^{-1}$ of $e^{+} p$ collision at a mean polarisation of $+0.32(-0.36)[3]$. The systematic uncertainty of these measurements, excluding the luminosity determination, was evaluated to be about $3 \%$, and is typically comparable or larger than that of the statistical uncertainty.

Figure 1 shows the measured cross sections as a function of the longitudinal polarisation of the $e^{ \pm}$beam. The figure also includes the HERA-I measurements of the unpolarised CC cross section [4]. The SM prediction, and its uncertainty due to PDFs as evaluated with the ZEUS-JETS PDFs, are also shown. The prediction passes zero at $P_{e}=-1(+1)$ for $e^{+}\left(e^{-}\right)$. The measurements are consistent with the SM prediction, clearly supporting the fully parity-violating nature (i.e., $V-A$ helicity structure) of the weak CC interaction. This is the first direct measurement showing the helicity dependence of the weak $\mathrm{CC}$ interaction at large energies.

\section{Combined EW and QCD analysis}

The cross section for NC DIS at HERA, $e^{ \pm} p \rightarrow e^{ \pm} X$, can be written as a ${ }^{\text {[5] }}$

$$
\frac{d^{2} \sigma_{N C}^{ \pm}\left(e^{ \pm} p\right)}{d x d Q^{2}}=\frac{2 \pi \alpha^{2}}{x Q^{4}}\left\{Y_{+}\left[F_{2}^{0, \pm}+P F_{2}^{P, \pm}\right]+Y_{-}\left[x F_{3}^{0, \pm}+P x F_{3}^{P, \pm}\right]\right\}
$$

where $\alpha$ is the electromagnetic fine structure constant and $Y_{ \pm}=1 \pm(1-y)^{2}$, with $y$ being the inelasticity. The structure functions $F_{2}^{0}$ and $x F_{3}^{0}$ are, for unpolarised $e^{ \pm} p$ collisions, defined as

$$
\begin{aligned}
F_{2}^{0, \pm} & =\Sigma x(q+\bar{q})\left[Q_{q}^{2}-2 v Q_{q} v_{q} K_{Z}+\left(v^{2}+a^{2}\right)\left(v_{q}^{2}+a_{q}^{2}\right) K_{Z}^{2}\right], \\
x F_{3}^{0, \pm} & =\Sigma x(q-\bar{q})\left[\mp 2 a Q_{q} a_{q} K_{Z} \pm 4 v a v_{q} a_{q} K_{Z}^{2}\right],
\end{aligned}
$$

\footnotetext{
${ }^{\text {a}}$ For simplicity, the longitudinal structure function $F_{L}$ is neglected in this formula.
} 
while, the structure functions $F_{2}^{P}$ and $x F_{3}^{P}$ are, for polarised $e^{ \pm} p$ collisions, defined as

$$
\begin{aligned}
F_{2}^{P, \pm} & =\Sigma x(q+\bar{q})\left[\mp 2 a Q_{q} v_{q} K_{Z} \pm 2 v a\left(v_{q}^{2}+a_{q}^{2}\right) K_{Z}^{2}\right], \\
x F_{3}^{P, \pm} & =\Sigma x(q-\bar{q})\left[2 v Q_{q} a_{q} K_{Z}-2\left(v^{2}+a^{2}\right) v_{q} a_{q} K_{Z}^{2}\right],
\end{aligned}
$$

where the sums run over quark flavours, $Q_{q}$ is the charge, $v_{q}$ and $a_{q}$ are the weak neutral vector and axial-vector couplings of the quark $q$. The kinematic factor $K_{Z}$ is defined as $K_{Z}=\frac{Q^{2}}{Q^{2}+M_{Z}^{2}} \frac{1}{\sin ^{2} 2 \theta_{W}}$ with $\theta_{W}$ being the Weinberg angle, and $v$ and $a$ are the vector and axial-vector coupling of the electron, given by $a=-\frac{1}{2}$ and $v=-\frac{1}{2}+2 \sin ^{2} \theta_{W}$, respectively. Equations (2), (3) and (4) illustrate that sensitivity to $a_{q}$ can be obtained from the unpolarised $\mathrm{NC}$ through the first term in $x F_{3}^{0,+}$, given that $x F_{3}^{0,+}$ can be accessed as the difference between $e^{+} p$ and $e^{-} p$ unpolarised cross sections. Similarly, equations (2), (5) and (6) illustrate that sensitivity to $v_{q}$ can be obtained from the polarised NC through the first term in $F_{2}^{P,+}$, given that $F_{2}^{P,+}$ can be accessed as the difference between positivelyand negatively-polarised cross sections. Hence, both the weak vector and axial-vector couplings of quarks can be determined by making use of HERA-I and HERA-II data together.

An EW and next-to-leading order QCD analysis of ZEUS data including NC and CC DIS cross sections measured with HERA-II polarised $e^{-}[6]$ was performed; by using $78.8(42.7) \mathrm{pb}^{-1}$ of luminosity at a mean polarisation of -0.27 $(+0.33)$ for CC [2] and 105.4 (71.8) $\mathrm{pb}^{-1}$ of luminosity at a mean polarisation of $-0.27(+0.30)$ for $\mathrm{NC}$ [7]. The analysis was done in the framework of our previous QCD analysis, ZEUSJETS, with addition of the polarised cross sections and of EW parameters to be also determined by the fit; $v_{u}, a_{u}, v_{d}, a_{d}$. These $4 \mathrm{EW}$ parameters were treated as free parameters in our fit, while other EW parameters, for example, $M_{Z}, M_{W}, G_{F}$, and $\sin \theta_{W}$ were fixed at the PDG values. The PDFs are parameterised at $Q_{0}^{2}=7 \mathrm{GeV}^{2}$ using the form $x f(x)=p_{1} x^{p_{2}}(1-$ $x)^{p_{3}}\left(1+p_{4}\right)$. The same constraints as in ZEUS-

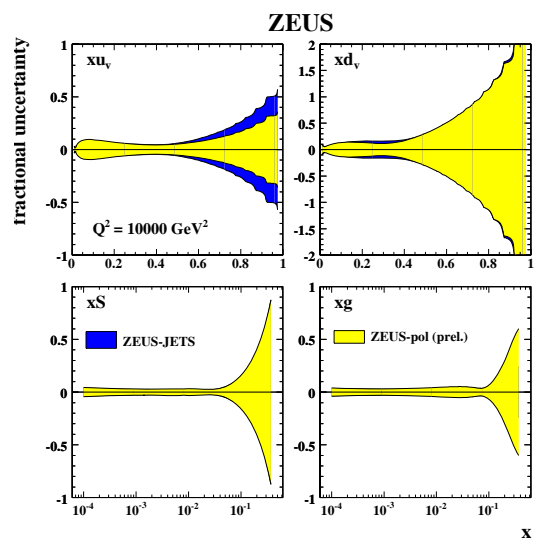

Figure 2: Fractional uncertainty of the PDFs determined by the combined EW and QCD analysis. JETS were applied to some of these parameters such that 11 parameters were left to describe the input PDFs. Thus, in total, 15 parameters of EW and PDFs are determined simultaneously. The uncertainty of the determined EW and PDF parameters due to the point-to-point correlated systematic uncertainties of experimental data was evaluated using the so-called OFFSET method [8].

Figure 2 shows the precision of the PDFs determined by the combined EW and QCD analysis compared with that determined by our previous QCD analysis of ZEUS-JETS. The precision of the high- $x$ PDFs is improved, particularly for the $u$-valence quark, since $e^{-} p$ data are dominated by $u$-quark at high $x$.

The left plot of Figure 3 shows the contour of $a_{u}$ vs $v_{u}$, and the right plot shows the contour of $a_{d}$ vs $v_{d}$. The figures also compare these contours to those determined by CDF 

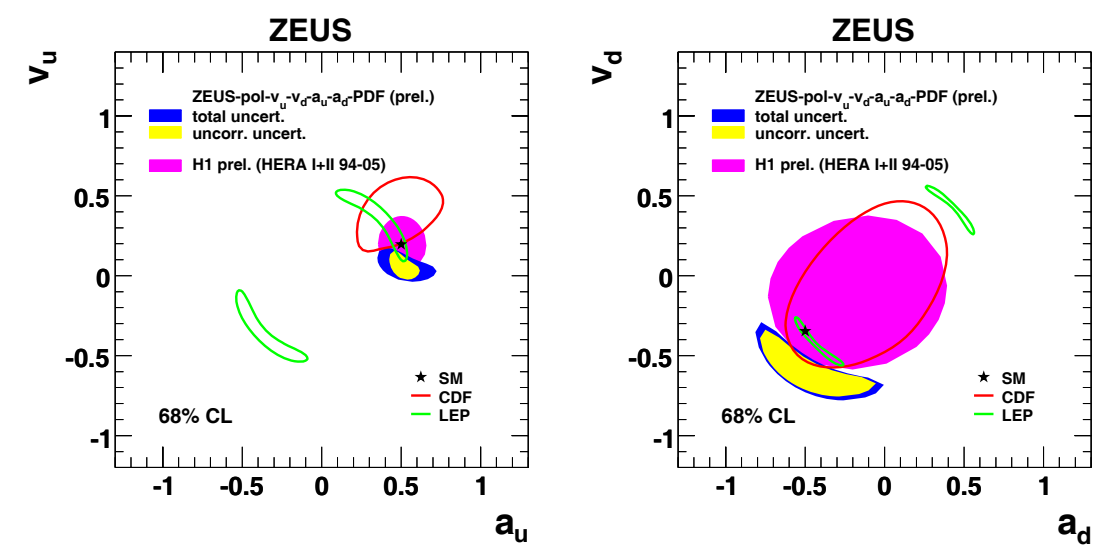

Figure 3: Determination of $v_{u}$ and $a_{u}$ (left plot) and $v_{d}$ and $a_{d}$ (right plot).

and LEP in which $a_{q}$ and $v_{q}$ are strongly correlated for each flavour $q$. In contrast, in our determination $a_{u}$ and $a_{d}$ are strongly correlated as they both enter into $x F_{3}^{0,+}$, and $v_{u}$ and $v_{d}$ are strongly correlated as they both enter into $F_{2}^{P,+}$. Therefore, HERA data provides a unique and complementary determination to CDF and LEP measurements. Furthermore, the precision is comparable or better compared to the other measurements. The $u$-quark couplings are much better determined than those of the $d$-quark, partly because the $d$-quark PDF itself is not so well determined. This situation is expected to improve as HERA-II $e^{+} p$ data are included in the analysis.

\section{Summary}

HERA data can now be used to examine the fundamental properties, and to determine the fundamental parameters, of the EW interaction. A combined EW and QCD analysis both on unpolarised and polarised DIS cross sections have determined the weak neutral couplings of quarks with precision comparable to or better than other experiments. Further more, improvements can be expected by making full use of all available data sets.

\section{References}

[1] ZEUS Coll., S. Chekanov et al., Eur. Phys. J. C42 1 (2005).

[2] ZEUS Coll., Contributed paper submitted to XXXIII International Conference on High Energy Physics.

[3] ZEUS Coll., Contributed paper \#86 submitted to The 2007 Europhysics Conference on High Energy Physics.

[4] ZEUS Coll., S. Chekanov et al., Phys. Lett. B539 197 (2002);

ZEUS Coll., S. Chekanov et al., Eur. Phys. J. C32 1 (2003).

[5] J. Bluemlein et al., Proc. of the HERA workshop 687 (1987).

[6] ZEUS Coll., Prelim. shown at XXIII Lepton-Phton 2007 Conference, August 13-18 Daegu, Korea.

[7] ZEUS Coll., Prelim. shown at XV International Workshop on Deep-Inelastic Scattering and Related Subjects, April 16-20, 2007, Munich, Germany.

[8] ZEUS Coll., S. Chekanov et al., Phys. Rev. D67 012007 (2003). 